\title{
Uso de plaguicidas inhibidores de acetilcolinesterasa en once entidades territoriales de salud en Colombia, 2002-2005
}

\author{
Omayda Cárdenas, Elizabeth Silva, Jaime Eduardo Ortiz ${ }^{\text {q.e.p.d. }}$
}

Grupo Salud Ambiental, Subdirección Red Nacional de Laboratorios, Instituto Nacional de Salud, Bogotá, D. C., Colombia

Institución donde se realizó el trabajo:

Entidades Territoriales de Salud y Grupo de Salud Ambiental, Subdirección Red Nacional de Laboratorios, Instituto Nacional de Salud, Bogotá, D.C., Colombia

Introducción. Por ser los plaguicidas anticolinesterásicos una causa importante de intoxicación y de muerte por intoxicación en los países en vía de desarrollo, el Instituto Nacional de Salud implementó el Programa de Vigilancia Epidemiológica de Organofosforados y Carbamatos, del cual, este informe corresponde al período 2002-2005.

Objetivo. Determinar la actividad de la acetilcolinesterasa en participantes con riesgo de exposición a plaguicidas organofosforados y carbamatos, e identificar los plaguicidas más utilizados en el área estudiada.

Materiales y métodos. Es un estudio descriptivo de información reportada al Programa de Vigilancia Epidemiológica de Organofosforados y Carbamatos por once departamentos colombianos, los cuales realizaron la determinación de acetilcolinesterasa con el equipo Lovibond.

Resultados. Participaron 28.303 personas con riesgo de exposición a plaguicidas; $81,4 \%$ eran hombres. El 9,3\% de las determinaciones analíticas fueron anormales, con una prevalencia de anormalidad entre los hombres de $9,9 \%$ y de $7,0 \%$ en las mujeres. Presentaron mayor prevalencia de valores anormales los grupos de edad de 18 a 25 años (12,3\%) y de 0 a 5 años (10,7\%), y los oficios de jornalero (27,0\%) y servicios generales del campo (26,1\%). De los 975 participantes, en el departamento del Meta, $80 \%$ de los participantes presentaron niveles anormales de actividad de la enzima. Los plaguicidas más reportados fueron los organofosforados $(39,7 \%)$ y carbamatos $(16,6 \%)$.

Conclusión. El incremento en la prevalencia de valores anormales de la acetilcolinesterasa y el riesgo de exposición infantil, hacen necesario disminuir el uso y la comercialización de plaguicidas de alto riesgo, y utilizar métodos menos tóxicos para el control de plagas.

Palabras clave: acetilcolinesterasa, plaguicidas, inhibidores de la acetilcolinesterasa, insecticidas organofosforados, carbamatos, exposición ocupacional.

The use of acetylcholinesterase inhibitors pesticides in eleven local health institutions, Colombia, 2002-2005

Introduction. Due to the importance of acetylcholinesterase inhibiting chemicals as pesticides in developing countries, the Instituto Nacional de Salud in Colombia designed the organophosphate and carbamate epidemiological surveillance program for the period 2002-2005.

Objective. The acetylcholinesterase activity was determined in study participants with a history of organophosphate and carbamate exposure and the most commonly used pesticides were identified in each study area.

Materials and methods. The information was compiled from reports sent to the Instituto Nacional de Salud organophosphate and carbamate epidemiological surveillance program from each of 11 provinces in Colombia. The analytical determination of the biomarker was performed by acetylcholinestare activity determined with the Lovibond field equipment.

Results. A total of 28,303 people were designated as having risk of exposure to pesticides. Most were men (81.4\%). Abnormal determinations averaged $9.3 \%$ (9.9\% in men and $7.0 \%$ in women). The $18-$ 25 year old age group showed the highest prevalence of abnormal results $(12.3 \%)$, followed by the group of $0-5$ year olds (10.7\%). The highest prevalence of abnormal acetylcholinesterase activity was in farm workers $(27.0 \%)$, followed by general outdoor activities (26.1\%). In the province of Meta, $80 \%$ of participants showed abnormal values of enzyme activity. The most commonly used pesticides were organophosphates $(39.7 \%)$ and carbamates $(16.6 \%)$.

Conclusion. The increase in the prevalence of abnormal values of acetylcholinesterase activity and the risk of exposure to pesticides in children necessitates a lowering of use and commercialization of high risk pesticides, and a need for developing safer methods for pest management. 
Key words: acetylcholinesterase, pesticides, cholinesterase inhibitors; organophosphates, insecticides; carbamates, occupational exposure.

Los plaguicidas son contaminantes ubicuos del medio ambiente, se han encontrado en aire, suelo, agua, plantas y en muestras de tejido humano y animal en todo el mundo. El uso continuo e inapropiado de los plaguicidas puede generar efectos biológicos adversos y eventos de "sobreexposición" en personas que se encuentran en contacto directo 0 indirecto con los agroquímicos. Estos efectos pueden ser causados, no sólo por el ingrediente activo y las impurezas asociadas, sino también por los solventes, vehículos, emulsificadores y otros constituyentes de los productos formulados (1-4). El amplio rango de compuestos usados en el control de plagas incluye insecticidas, fungicidas, herbicidas, rodenticidas y molusquicidas, entre otros; los más usados a nivel mundial son los insecticidas y, entre éstos, los organoclorados, organofosforados y carbamatos (5).

La exposición a plaguicidas es un tema global de salud pública. En 1972, la Organización Mundial de la Salud calculó que cada año ocurría medio millón de envenenamientos en el mundo con más de 5.000 defunciones; en la siguiente década estimó en más de tres millones las intoxicaciones, con una mortalidad probable de 1\% (6). La mayoría de estos hechos ocurren en países en desarrollo (7), en donde la mayor parte de las muertes ocasionadas por plaguicidas son el resultado de la exposición a organofosforados y carbamatos (8). La alta incidencia de intoxicaciones y muertes que se presentan anualmente por plaguicidas ha hecho que en los países de América Latina y el Caribe estos productos se consideren como un problema grave de alto impacto en la salud pública (9). Las Naciones Unidas, considerando que la tasa de intoxicación en los países en desarrollo podría ser 13 veces mayor que en los países industrializados, declaró a los plaguicidas como uno de los mayores problemas en el ámbito mundial (6).

Se ha estimado que al sector agropecuario corresponde cerca de $85 \%$ del consumo de plaguicidas en el mundo (10) y, según publicaciones

\footnotetext{
Correspondencia:

Omayda Cárdenas, Grupo de Salud Ambiental, Subdirección Red Nacional de Laboratorios, Instituto Nacional de Salud, Avenida calle 26 № 51-20, Bogotá, D.C., Colombia.

Teléfono: (571) 220 7700, extensiones 448 y 449; fax: (571) 220

7700 , extensión 447

ocardenas@ins.gov.co

Recibido: 24/04/09; aceptado:26/10/09
}

de la Organización Internacional del Trabajo (OIT), el envenenamiento por plaguicidas estaría ocasionando $14 \%$ de las lesiones ocupacionales del sector agrícola (11). Una de las posibles causas de esta situación es la deficiente información que se les suministra a los trabajadores agrícolas sobre los riesgos que conlleva la manipulación y aplicación de plaguicidas y, también, el que ellos no tomen sus propias medidas de protección durante la exposición laboral $(8,12,13)$. En Colombia, según los datos de la Encuesta Nacional Agropecuaria de $2004,44,8 \%$ de la superficie del país estuvo destinada a los sectores agrícola y pecuario; la agricultura participó con $7,2 \%$ de la superficie agropecuaria, es decir, 3'708.455 hectáreas. En este uso los cultivos permanentes participaron con $51,3 \%$ del área agrícola, los cultivos transitorios y de barbecho con $45,9 \%$ y el área de descanso con 2,8\% (14).

En Colombia, la industria de plaguicidas se inició hacia 1962 con el proceso de formulación y, posteriormente, se procedió con la síntesis de algunos ingredientes activos. En los últimos treinta años, las formulaciones de plaguicidas registradas en el Instituto Colombiano Agropecuario prácticamente se han duplicado, pasando de 770 productos en 1974 (formulados con base en 186 ingredientes activos), a 1.370 en el 2003, formulados con base en 400 ingredientes activos (6). La comercialización de los agroquímicos estuvo en incremento, tanto en lo referente a la producción como a la venta de plaguicidas. Durante los años correspondientes al presente informe, la mayor cantidad de productos estuvo representada por herbicidas, fungicidas e insecticidas; de estos últimos, los organofosforados y carbamatos representaron más de $80 \%$ de la comercialización $(15,16)$.

Los organofosforados y carbamatos son inhibidores de la colinestera, enzima humana que cataliza la hidrólisis de los ésteres del neurotransmisor acetilcolina. Los organofosforados se unen a la acetilcolinesterasa e inhiben su actividad por fosforilación irreversible, tanto en los glóbulos rojos como en el plasma, por lo cual su restauración depende de la síntesis de nuevas moléculas de la enzima, a diferencia de los carbamatos en los que esta unión es reversible de manera espontánea $(7,8,17)$. La absorción de los agentes anticolinesterásicos ocurre por inhalación, por ingestión y a través de la piel, y puede ser detectada 
determinando la actividad de la acetilcolinesterasa como biomarcador para evaluar individuos o grupos de población con riesgo de exposición a plaguicidas organofosforados y carbamatos. En un contexto ocupacional, la medición de la actividad de la enzima puede proporcionar un medio para evaluar el adecuado uso de las medidas de protección y seguridad en el lugar de trabajo $(5,8,17)$.

La colinesterasa plasmática es inhibida más rápidamente que la eritrocítica y sus niveles normales se restablecen dentro de los 60 días posteriores a la exposición, siendo un indicador de intoxicación aguda por organofosforados. La actividad de la acetilcolinesterasa eritrocítica se deprime más lentamente y toma varias semanas y hasta meses para retornar a niveles normales; por lo tanto, es la medida de elección y de importancia en los sistemas de vigilancia para evaluar la exposición crónica a plaguicidas anticolinesterásicos (7). Este seguimiento ofrece la oportunidad de identificar los trabajadores con "sobreexposición" y tomar las medidas indicadas antes de que la toxicidad se manifieste clínicamente.

Para una correcta evaluación e interpretación de los resultados de laboratorio, es conveniente precisar condiciones especiales que modifican los niveles de la colinesterasa plasmática, principalmente. Se encuentra reducción en la actividad de la enzima durante el embarazo y la menstruación, y en anemias, quemaduras, desnutrición o cáncer; se incrementa en condiciones de alcoholismo, diabetes, artritis, hiperlipidemia y obesidad, entre otras (4).

El Ministerio de Salud, mediante el Decreto 1843 de 1991 (18), estableció que las seccionales de salud debían desarrollar un programa específico de vigilancia epidemiológica de plaguicidas, con notificación obligatoria de los casos de intoxicación o de accidente a causa de estos productos. Entre 2003 y 2005, fueron reportados al Sistema de Vigilancia en Salud Pública, SIVIGILA, 2.950 casos en promedio por año (19). Además, la Resolución 4547 de 1998 (20) planteó que el Programa de Vigilancia Epidemiológica de organofosforados y carbamatos (VEO) realizara el seguimiento y control de estos plaguicidas, determinando la actividad de la acetilcolinesterasa.

Dando cumplimiento a la norma, en el Instituto Nacional de Salud se lleva a cabo dicho programa, el cual está dirigido a trabajadores ocupacionalmente expuestos y a personas con riesgo de exposición ambiental por permanecer cerca de los sitios de aplicación de plaguicidas. Informa sobre los plaguicidas más utilizados según el reporte de los trabajadores y de las intoxicaciones producidas por los plaguicidas anticolinesterásicos, con el propósito de impulsar el desarrollo de acciones de promoción, prevención y control, que contribuyan con la disminución del efecto de estos tóxicos sobre la salud (21-23).

\section{Materiales y métodos}

Se presenta un estudio descriptivo con base en la información reportada por los departamentos colombianos que utilizan plaguicidas y que participan voluntariamente en el Programa de Vigilancia Epidemiológica, VEO. Para el desarrollo del programa se realizan convenios entre el Instituto Nacional de Salud y las entidades territoriales de salud, las cuales llevan a cabo un inventario de las empresas, censo de las personas ocupacional y ambientalmente expuestas a plaguicidas, y entrevistas, informando sobre los objetivos, importancia y procedimiento del programa. A las empresas que voluntariamente deciden participar, se les realiza una encuesta con el fin de registrar la información con sus datos generales y de funcionamiento. Igualmente, a los trabajadores que mediante consentimiento informado aceptan participar, se les aplica un formulario con variables demográficas, ocupacionales, de protección personal y de exposición, y se anota el resultado de la actividad de la acetilcolinesterasa, el cual ha sido obtenido después de realizar la toma de la muestra de sangre y la determinación analítica respectiva.

La información obtenida es digitada por cada entidad territorial de salud en un formato previamente diseñado por el Instituto Nacional de Salud en el programa Epi-Info. Los formatos son enviados en medio magnético o por correo electrónico al Laboratorio Nacional de Referencia, Grupo de Salud Ambiental del Instituto Nacional de Salud, responsable de la parte complementaria del programa, la cual comprende, además del análisis de la información y divulgación de los resultados, las actividades correspondientes a la supervisión del programa, la realización de capacitaciones y visitas técnicas, y el desarrollo del Programa Interlaboratorios de Control de Calidad.

\section{Población muestra}

Hicieron parte del estudio personas directamente expuestas a plaguicidas como resultado de su ocupación, y otras indirectamente expuestas por habitar o laborar en sitios cercanos a la 
aplicación de los agroquímicos. La participación en el programa fue voluntaria y los menores de edad fueron incluidos con el previo consentimiento informado de los padres.

\section{Determinación analítica}

La medición del porcentaje de actividad de la acetilcolinesterasa se realizó por el método colorimétrico y semicuantitativo de Limperos y Ranta, modificado por E. F. Edson (24) y utilizando el equipo de campo Lovibond. Este equipo permite realizar determinaciones analíticas con una pequeña cantidad de sangre total, de manera rápida, con alta especificidad, con precisión y bajo costo (25-27).

El método se fundamenta en un cambio de $\mathrm{pH}$, detectado como cambio de color en un tiempo determinado y expresado en porcentaje de actividad, en valores discretos y a intervalos de $12,5 \%$. Se consideran anormales los valores menores al $75 \%$ de actividad de colinesterasa. El procedimiento se encuentra descrito en las anteriores publicaciones del Programa VEO $(22,23)$. Para evaluar el desempeño de los laboratorios participantes, se aplica el Programa Interlaboratorios de Control de Calidad, coordinado por el Grupo de Salud Ambiental de la Subdirección Red Nacional de Laboratorios.

\section{Análisis de la información}

Se realizó un análisis descriptivo mediante frecuencias simples, tanto para la población total como para los participantes con niveles anormales de actividad de acetilcolinesterasa, para hacerlo, se empleó el programa Epi Info 6.04 (Centers for Disease Control and Prevention (CDC), USA).

\section{Resultados}

Durante el período comprendido entre el 2002 y el 2005, reportaron información al Programa VEO once entidades territoriales de salud, con un total de 28.303 participantes con riesgo de exposición a plaguicidas (cuadro 1).

Los laboratorios de salud pública de las once entidades territoriales de salud participaron en la evaluación del desempeño del Programa Interlaboratorios de Control de Calidad y los resultados se evaluaron teniendo en cuenta la media corregida, más o menos 1 o 2 desviaciones estándar (DE). El desempeño fue catalogado como excelente para cinco entidades territoriales de salud y aceptable para las demás (figura 1).

\section{Características generales y ocupacionales de los participantes}

De los 28.026 participantes que informaron el oficio, $80,8 \%$ (22.649) estaban directamente expuestos y $19,2 \%$ (5.377) indirectamente expuestos. Los oficios de exposición directa con mayor número de trabajadores fueron: fumigador-aplicador $(38,2 \%)$, jornalero $(24,9 \%)$ y servicios generales del campo $(16,3 \%)$. Las personas que no laboraban con plaguicidas, pero se encontraban en el área de influencia de los agroquímicos, se distribuyeron así: niños en edad escolar, 33,0\%; amas de casa, niños no escolares y mayores de 60 años, 29,5\%; personal administrativo, 27,3\%, y profesionales sin exposición laboral a plaguicidas, $10,2 \%$.

Cuadro 1. Número de participantes con riesgo de exposición a plaguicidas por año y entidad territorial de salud, 2002-2005.

\begin{tabular}{|c|c|c|c|c|c|c|c|c|c|c|}
\hline \multirow{2}{*}{$\begin{array}{l}\text { Entidad } \\
\text { territorial } \\
\text { de salud }\end{array}$} & \multicolumn{2}{|c|}{2002} & \multicolumn{2}{|c|}{2003} & \multicolumn{2}{|c|}{2004} & \multicolumn{2}{|c|}{2005} & \multicolumn{2}{|c|}{ Total trabajadores } \\
\hline & $\mathbf{n}$ & $\%$ & $\mathbf{n}$ & $\%$ & $\mathbf{n}$ & $\%$ & $\mathbf{n}$ & $\%$ & $n$ & $\%$ \\
\hline Boyacá & 0 & 0,0 & 132 & 0,5 & 0 & 0,0 & 134 & 0,5 & 266 & 0,9 \\
\hline Caldas & 403 & 1,4 & 318 & 1,1 & 1.742 & 6,2 & 1.319 & 4,7 & 3.782 & 13,4 \\
\hline Córdoba & 0 & 0,0 & 0 & 0,0 & 690 & 2,4 & 0 & 0,0 & 690 & 2,4 \\
\hline Guaviare & 0 & 0,0 & 0 & 0,0 & 0 & 0,0 & 117 & 0,4 & 117 & 0,4 \\
\hline Huila & 2.601 & 9,2 & 25 & 0,1 & 212 & 0,7 & 724 & 2,6 & 3.562 & 12,6 \\
\hline Meta & 0 & 0,0 & 0 & 0,0 & 975 & 3,4 & 0 & 0,0 & 975 & 3,4 \\
\hline Nariño & 0 & 0,0 & 107 & 0,4 & 250 & 0,9 & 250 & 0,9 & 607 & 2,1 \\
\hline Quindío & 0 & 0,0 & 178 & 0,6 & 304 & 1,1 & 0 & 0,0 & 482 & 1,7 \\
\hline Santander & 849 & 3,0 & 0 & 0,0 & 0 & 0,0 & 2.458 & 8,7 & 3.307 & 11,7 \\
\hline Sucre & 0 & 0,0 & 293 & 1,0 & 1.059 & 3,7 & 344 & 1,2 & 1.696 & 6,0 \\
\hline Valle del Cauca & 3.111 & 11,0 & 845 & 3,0 & 4.681 & 16,5 & 4.182 & 14,8 & 12.819 & 45,3 \\
\hline Totales & 6.964 & 24,6 & 1.898 & 6,7 & 9.913 & 35,0 & 9.528 & 33,7 & 28.303 & 100,0 \\
\hline
\end{tabular}

Fuente: bases de datos reportadas por las entidades territoriales de salud, Programa VEO, 2002-2005, Grupo de Salud Ambiental, Subdirección Red Nacional de Laboratorios 


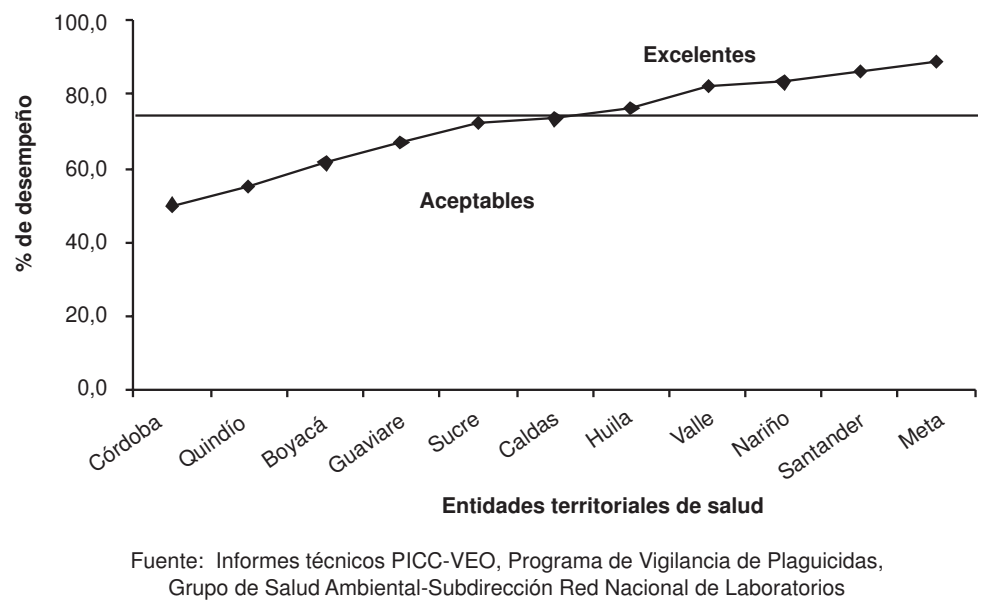

Figura 1. Programa Interlaboratorios de Control de Calidad, PICC-VEO. Evaluación del desempeño 2004-2005

Según la información reportada por los 28.164 participantes, $81,4 \%$ (22.926) eran de sexo masculino y $18,6 \%$ (5.238) de sexo femenino. De las 3.438 mujeres que informaron sobre la variable de estado de embarazo, $12,3 \%$ (421) se encontraban en estado de gravidez. El rango de edad con mayor número de participantes fue el de 26 a 40 años, con 40,5\% (11.048), seguido por el de 41 a 50 años, con 18,9\% (5.145), y el de 18 a 25 años, con 18,0\% (4.851); el promedio de edad fue de 35,6 años (rango, 1 a 92 años; $D E=13,5$ ).

De los 26.654 participantes que reportaron información sobre afiliación al Sistema General de Seguridad Social en Salud, 76,2\% (20.310) contaba con algún tipo de seguridad en salud; de ellos, $52,4 \%(10.640)$ pertenecía al régimen contributivo y el $47,6 \%$ restante, al régimen subsidiado. De los pertenecientes al régimen contributivo, 43,4\% (4.613) refirió afiliación a riesgos profesionales.

\section{Exposición y uso de plaguicidas}

Según la información reportada por 23.755 participantes, 95,1\% (22.588) estuvo expuesto alguna vez a plaguicidas; el promedio del tiempo de exposición fue de 65,5 meses (mínimo, 1, y, máximo, 680; $D E=86,1)$. En el cuadro 2 se encuentra el listado de los plaguicidas por grupo químico de acuerdo con la clasificación planteada por el Ministerio de la Protección Social (11) y corresponde a los plaguicidas que los trabajadores reportaron como los más frecuentemente usados en sus actividades laborales.

Al revisar la clasificación por categoría toxicológica, lamás empleadafue lacategoríalen 35,2\%(30.271), seguida por la III, en 30,2\% (25.909), categoría II en $23,4 \%$ (20.095) y categoría IV en 11,2\% (9.647). En relación con el tipo de organismo por controlar, $68,5 \%$ (58.863) eran insecticidas, 15,5\% (13.350) herbicidas, $15,4 \%$ (13.274) fungicidas y $0,5 \%$ (435) fertilizantes o desinfectantes.

El uso más frecuente de los plaguicidas fue en el área agrícola, reportado por $91,9 \%$ de los participantes. De los 43.269 registros informados, los cultivos más frecuentes en los que se aplicaron los plaguicidas fueron: arroz (15,3\%), café $(11,7 \%)$, tomate $(10,1 \%)$ y maíz $(5,4 \%)$. El restante $8,1 \%$ de los participantes reportó el uso de plaguicidas en campañas sanitarias de salud, área doméstica, aplicación a productos almacenados y sector pecuario.

En cuanto a la protección personal, según la información suministrada por 16.742 participantes,

Cuadro 2. Plaguicidas más usados por los trabajadores, según grupo químico, 2002-2005.

\begin{tabular}{lcc}
\hline Grupo químico & Frecuencia & Porcentaje \\
\hline Organofosforados & 34.143 & 39,7 \\
Carbamatos & 14.265 & 16,6 \\
Organoclorados & 3.063 & 3,6 \\
Otros compuestos clorados & 6.512 & 7,6 \\
(clorinados) & & \\
Piretroides & 4.286 & 5,0 \\
Etilenbisditiocarbamatos & 9.222 & 10,7 \\
Bipiridilos & 1.550 & 1,8 \\
Otros & 6.145 & 7,2 \\
Ácido fosfónico & 6.736 & 7,8 \\
Total & 85.922 & 100,0 \\
\hline
\end{tabular}

Fuente: bases de datos reportadas por las entidades territoriales de salud, Programa VEO, 2002-2005, Grupo de Salud Ambiental, Subdirección Red Nacional de Laboratorios 
$38,2 \%$ (6.400) usaba algún elemento de protección personal; los más frecuentemente reportados fueron overol y botas $(20,6 \%)$, seguido por el uso de respirador o tapabocas, guantes y overol $(15,0 \%)$, únicamente tapabocas $(13,8 \%)$, botas $(12,5 \%)$ y overol $(11,6 \%)$.

De los 17.041 participantes que respondieron las preguntas relacionadas con capacitación, 70,3\% (11.985) informó haber recibido capacitación sobre el uso y manejo de plaguicidas en el último año; el restante $29,7 \%$ manifestó no haber tenido ningún tipo de capacitación sobre el uso de plaguicidas en sus labores diarias.

\section{Pruebas de actividad de acetilcolinesterasa}

Se realizaron 28.303 determinaciones analíticas para medir el porcentaje de actividad de la acetilcolinesterasa, una por cada participante con riesgo de exposición a plaguicidas. Del total de pruebas, $81,5 \%$ (23.067) se hicieron en trabajadores que refirieron haber estado expuestos a plaguicidas en los 30 días previos a la toma de la muestra de sangre y $18,5 \%$ (5.236), en participantes que manifestaron no haber estado expuestos a este tipo de plaguicidas en el último mes.

El 9,3\% (2.628) de los participantes presentó porcentajes de actividad de acetilcolinesterasa menores o iguales a $62,5 \%$, valores considerados como anormales. La figura 2 presenta la distribución por año del número de pruebas de actividad de acetilcolinesterasa realizadas de 2002 a 2005.

Características generales y ocupacionales de los participantes con actividad anormal de acetilcolinesterasa

Se presentaron valores anormales de acetilcolinesterasa en $9,9 \%$ (2.263) de los hombres participantes en el estudio y en 7,0\% (365) de las mujeres; de ellas, cerca de $70 \%$ se encontraba en edad fértil. El grupo de edad con mayor prevalencia de valores anormales fue el de 18 a 25 años (12,3\%), seguido por el de 0 a 5 años (10,7\%).

Los oficios mayormente practicados por los 2.597 trabajadores con valores anormales, que proporcionaron información sobre esta variable, fueron: jornalero, 27,3\% (709), servicios generales del campo, 26,4\% (685), y fumigador-aplicador, 24,2\% (629). El 6,8\% (364) de los participantes indirectamente expuestos presentaron valores anormales de actividad de acetilcolinesterasa, entre los cuales $57,4 \%$ (209) fueron niños en edad escolar, niños menores de 5 años, amas de casa y personas mayores de 60 años.

De los 2.481 participantes con niveles anormales de acetilcolinesterasa, que reportaron información sobre afiliación al Sistema General de Seguridad Social en Salud, 79,8\% (1.981) tenía algún tipo de seguridad social en salud; de éstos, $86,3 \%$ (1.710) refirió afiliación al régimen contributivo y $13,7 \%$ (271) al régimen subsidiado; el restante $20,2 \%$ de los participantes no contaba con seguridad social en salud.

El departamento con mayor prevalencia de valores anormales de acetilcolinesterasa fue el Meta: el $80 \%$ de sus participantes presentó niveles anormales en la actividad de la enzima. El Valle del Cauca aportó el $45 \%$ de los participantes en el estudio y tuvo un $4,0 \%$ de valores anormales.

\section{Exposición y uso de plaguicidas}

Según indagación realizada a los participantes sobre si estuvieron expuestos a plaguicidas en los 30 días previos a la determinación de la

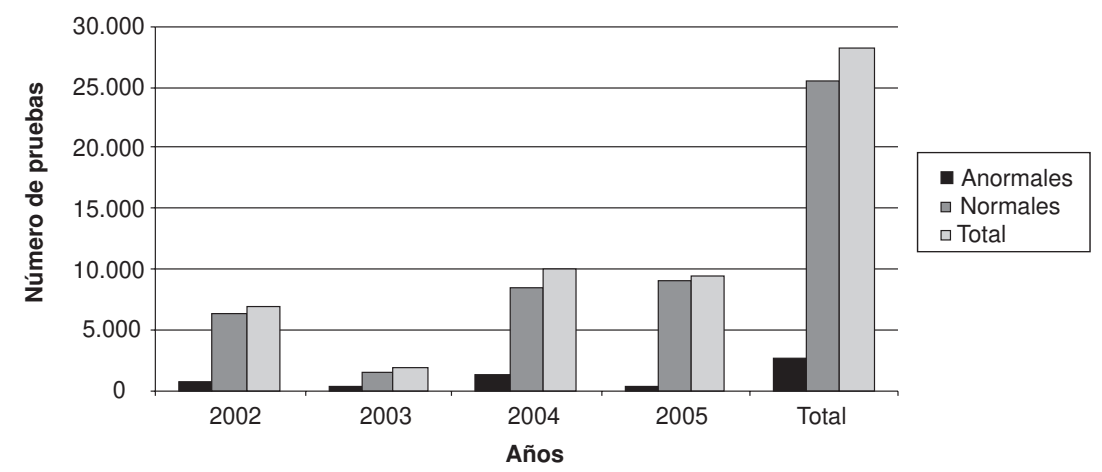

Fuente: bases de datos reportadas por las ETS, Programa VEO 2002-2005, Grupo de Salud Ambiental-Subdirección Red Nacional de Laboratorios

Figura 2. Pruebas de acetilcolinesterasa realizadas a los participantes en el estudio, 2002-2005 
actividad de la acetilcolinesterasa, de los 23.067 que respondieron afirmativamente, 7,7\% (1.777) presentó valores anormales de actividad de la enzima; de éstos, 99,4\% refirió haber estado expuesto a plaguicidas organofosforados, a carbamatos o ambos, en los últimos 30 días. La exposición ocupacional de estos trabajadores estuvo referida a actividades agrícolas en $89,7 \%$ de los casos y a actividades sanitarias en los casos restantes. De estas últimas, 60,7\% fueron en el área doméstica, $25,6 \%$ en campañas sanitarias y $13,6 \%$ en productos almacenados y en el sector pecuario. Sólo 14,4\% (256) de los trabajadores reportó el uso de botas, overol y botas, y tapabocas y guantes, como elementos de protección personal.

De los 5.263 que refirieron no haber estado expuestos a plaguicidas en el mes previo a la toma de la muestra, 851 (16,2\%) presentaron valores de acetilcolinesterasa menores o iguales a $62,5 \%$. Al preguntarles si alguna vez habían estado expuestos a plaguicidas, 98,8\% (841) respondió haber tenido exposición laboral durante 8,4 años en promedio, principalmente en actividades agrícolas. De ellos, sólo 2,1\% (18) reportó haber usado guantes, botas, tapabocas u overol, como elementos de protección personal.

Los trabajadores con valores anormales de acetilcolinesterasa reportaron el uso de 5.208 plaguicidas; su frecuencia y clasificación según grupo químico se encuentra en la cuadro 3. Al analizar la clasificación por categoría toxicológica, el primer lugar fue para la categoría I con 36,9\% (1.923), seguida por la categoría III con $28,3 \%$ (1.475), la categoría II con $19,0 \%$ (992) y la categoría IV con $15,7 \%$ (818). Los plaguicidas usados según el organismo por controlar fueron: insecticidas, 65,1\% (3.393), herbicidas, 20,6\% (1.074) y fungicidas, $12,7 \%$ (660). Los principales cultivos en los que se aplicaron los plaguicidas reportados por los trabajadores con valores anormales de acetilcolinesterasa, fueron: palma de aceite, 30,6\% (1.097), tomate, 10,3\% (371), y arroz, 7,0\% (250).

Las medidas correctivas aplicadas a la mayor parte de los participantes con valores anormales de acetilcolinesterasa fueron: $38,2 \%$ (1.004) fue retirado de la exposición a plaguicidas, 10,1\% (266) fue retirado de la exposición y remitido al médico, a $9,6 \%$ (253) se le repitió la prueba a los 15 días pero no se retiró de la exposición, a 9,6\% (252) se le retiró de la fuente de exposición a plaguicidas y se le repitió la prueba a los 15 días, y a 24,9\% (654)
Cuadro 3. Plaguicidas usados por los participantes que presentaron niveles anormales de acetilcolinesterasa, según grupo químico, 2002-2005.

\begin{tabular}{lcc}
\hline Grupo químico & Frecuencia & Porcentaje \\
\hline Organofosforados & 1.959 & 37,6 \\
Carbamatos & 912 & 17,5 \\
Organoclorados & 153 & 2,9 \\
Otros compuestos & 359 & 6,9 \\
clorados (clorinados) & & \\
Piretroides & 210 & 4,0 \\
Etilenbisditiocarbamatos & 484 & 9,3 \\
Bipiridilos & 148 & 2,8 \\
Otros & 410 & 7,9 \\
Ácido fosfónico & 573 & 11,0 \\
Total & 5.208 & 100,0 \\
\hline
\end{tabular}

Fuente: bases de datos reportadas por las entidades territoriales de salud, Programa VEO, 2002-2005, Grupo de Salud Ambiental, Subdirección Red Nacional de Laboratorios

no se le aplicó ninguna medida correctiva. Muy pocos trabajadores (437) refirieron haber recibido capacitación en el uso y manejo de plaguicidas en el último año.

\section{Discusión}

En el presente estudio, los oficios con mayor prevalencia de valores anormales de actividad de acetilcolinesterasa fueron los de mayor riesgo de exposición: jornalero, servicios generales de campo y fumigador-aplicador, con edades comprendidas entre los 18 y los 25 años, las cuales corresponden a la población joven, económicamente activa. Estos resultados son iguales a los encontrados en el programa VEO de años anteriores (23).

Aunque las mujeres dedicadas a la actividad de la agricultura presentaron un porcentaje menor $(4,8 \%)$ que el reportado para Costa Rica $(8,0 \%)$ (28), las accidentalmente expuestas representaron el $13,8 \%$ de la población estudiada, cifras relevantes considerando las condiciones fisiológicas de vulnerabilidad para la salud y los efectos adversos para la reproducción.

Es importante tener en cuenta que los niños han ocupado el segundo lugar con respecto a los grupos de edad con mayor prevalencia de valores anormales de acetilcolinesterasa, tanto en el presente estudio como en informes anteriores del Programa VEO (23). En América Latina, las familias campesinas trabajan con los niños en los campos, se hacen las aplicaciones de plaguicidas en su presencia, almacenan estos tóxicos en sus casas, e incluso, ayudan a los adultos en estas tareas (29); específicamente, en Nicaragua se reportó 
que, durante la década de los 90 , entre $10 \%$ y $20 \%$ de las intoxicaciones habían ocurrido en menores de quince años; llama la atención que los niños no deberían estar expuestos ocupacionalmente a los plaguicidas (30).

Para tratar de controlar esta situación, habría que implementar un trabajo intersectorial con la participación de los diferentes ministerios y la población organizada. Este es un problema que no se puede resolver solamente con las leyes, sino mediante un nuevo modelo de salud familiar que prevea la participación de las comunidades y el fortalecimiento de los instrumentos ya existentes. Los niños son más vulnerables que los adultos a los tóxicos, debido a que los químicos que alteran el comportamiento celular son más activos en los organismos infantiles por encontrarse en etapa de crecimiento. Los plaguicidas organofosforados afectan el comportamiento del sistema nervioso central todavía poco desarrollado en los niños, y disminuyen la coordinación psicomotriz y la capacidad de concentración $(17,29)$.

Según la toxicidad los plaguicidas, pueden afectar el crecimiento, la supervivencia por alteración en los factores reproductivos y, en casos de toxicidad aguda, pueden llegar a causar la muerte (31).

El alto porcentaje de valores anormales de acetilcolinesterasa encontrado en el departamento del Meta, requiere estudios de tipo analítico en el que se evalúen otros factores de riesgo que puedan estar influyendo en dicho resultado. Por lo tanto, se analizará la evolución de los valores de acetilcolinesterasa en la información reportada en los años posteriores al presente estudio.

La mayoría de plaguicidas reportados por los participantes en el estudio están catalogados como alta y moderadamente tóxicos, y pueden causar intoxicaciones agudas y efectos adversos sobre la salud a largo plazo. Además, los de categoría toxicológica III y IV no pueden ser catalogados como inocuos para la salud, teniendo en cuenta, entre otros aspectos, que los participantes han estado expuestos a ellos de manera continua y durante períodos prolongados.

Es importante señalar que la población participante en el estudio ha tenido un tiempo promedio de exposición a plaguicidas de 5,5 años y, haciendo referencia al tiempo máximo de exposición, algunos de los trabajadores refirieron que "toda la vida" han estado laborando con plaguicidas. Ante este tipo de exposiciones a largo plazo, los plaguicidas se han relacionado con procesos de mutagénesis somática y germinal (31), riesgos críticos a los que estarían expuestos tanto los trabajadores participantes como sus familias.

Con respecto a los largos períodos de exposición, llama la atención que el grupo de participantes que manifestó no haber estado expuesto a plaguicidas 30 días antes de la toma de la muestra para la determinación de la acetilcolinesterasa, presentó una mayor prevalencia de valores anormales de actividad de la enzima que los expuestos en ese período, y manifestó haber tenido 8,4 años de exposición a plaguicidas en promedio. Habría que determinar si estos trabajadores, al estar sometidos a repetidas exposiciones a plaguicidas anticolinesterásicos, presentan de manera crónica valores bajos de actividad de la acetilcolinesterasa, los cuales no alcanzan a recuperarse antes de la siguiente exposición, aunque ésta suceda en un período superior a los 30 días de la anterior exposición.

Los plaguicidas organofosforados presentan riesgos de exposición conocidos en términos de afecciones a la salud e índices de morbilidad; actualmente, son los causantes del mayor número de intoxicaciones ocasionadas por agroquímicos, tanto a nivel nacional (21-23,32-34) como internacional $(7,8,12,35,36)$. Este tipo de plaguicida fue el más reportado por los trabajadores en el presente estudio $(39,6 \%)$; esto también fue lo encontrado en el estado de Nueva York, durante el período de 1990 a 1993, con $71 \%$ de reporte de uso por parte de 13.417 aplicadores de plaguicidas (7).

A nivel mundial, los organofosforados han ganado popularidad y preferencia sobre otro tipo de plaguicidas, porque son menos persistentes y causan menos impacto en el medio ambiente. Sin embargo, algunos de ellos han sido restringidos por la Environmental Protection Agency (EPA) de los Estados Unidos, en orden a salvaguardar la salud del hombre y de los animales y a proteger el medio ambiente (7). El 58,6\% del total de organofosforados reportados en este estudio se encuentran en el listado de plaguicidas de uso restringido por la EPA. Entre ellos están el clorpirifos, con un 25,6\% (8.756) de los reportes, el metamidofos, con $19,5 \%$ (6.643), el profenofos, con $5,5 \%$ (1.870), el metil-paratión (que también se encuentra restringido en Colombia), con $4,7 \%$ (1.611), el fenitrotión, con 2,3\% (777), y el diazinón, con $0,9 \%(291)$. 
Entre los 1.959 organofosforados reportados por los trabajadores que presentaron niveles anormales de acetilcolinesterasa, 30,2\% (591) era clorpirifos, $19,6 \%$ (383), metamidofos, 3,6\% (71), profenofos, 6,7\% (132), metil-paratión, 0,4\% (8), fenitrotión, y 0,3\% (7), diazinón. De estos plaguicidas, los de mayor porcentaje de reporte coinciden con los informados por el Proyecto de Aspectos Ocupacionales y Ambientales de la Exposición a Plaguicidas en el Istmo Centroamericano (Plagsalud) como los responsables de la mayor morbimortalidad en estos países (37). El clorpirifos, que fue el organofosforado más reportado por los trabajadores, es uno de los insecticidas de mayor uso en el mundo; sus posibles efectos de exposición han sido ampliamente estudiados, y se ha encontrado que su grupo fosforilo es un sitio potencialmente electrofílico y su acción se caracteriza por la inhibición de la síntesis del ADN (31).

Un porcentaje considerable de participantes refirió el uso de carbamatos, grupo químico reportado por los participantes con valores anormales de acetilcolinesterasa en $17,5 \%$, similar a los valores informados por el sistema de vigilancia en salud pública de las intoxicaciones por plaguicidas en El Salvador, durante el período de 1996 al 2002, en el que se observó una variación de 18,9\% a 21,9\% (36). Además, varios participantes reportaron haber estado expuestos a ambos tipos de plaguicidas inhibidores de la acetilcolinesterasa.

Con respecto a los organoclorados, se encontraron en uso los de tipo endosulfán $(3,6 \%)$ y, aunque su uso fue prohibido en Colombia en el 2001 (38), se ha reportado que estos productos entran por contrabando al país $(39,40)$. Sin embargo, es importante destacar que el endosulfán ha tenido una disminución considerable en su reporte de uso, el cual fue de $8,4 \%$ en el periodo de 1998 a 2001 según informe del VEO (23). Posiblemente, los organoclorados han sido reemplazados por otros compuestos clorados, cuyo empleo subió de $6,6 \%$ a $7,6 \%$ comparando con el reporte anteriormente citado. Aunque es una situación favorable desde el punto de vista de la toxicidad, hay que tener en cuenta que, debido al contenido de moléculas de cloro en la composición química, algunos compuestos clorados se catalogan como plaguicidas de alto riesgo.

Otro grupo químico en el que se encontró incrementado el reporte de uso fue el ácido fosfónico. Varió de $2,3 \%$ en el informe del VEO del período 1998-2001 (23) a 7,8\% en el presente estudio, quedando como el cuarto tipo de plaguicida utilizado según el grupo químico. Los plaguicidas contemplados en este grupo químico, generalmente, son los que tienen como ingrediente activo el glifosato. Estos herbicidas se consideran en la literatura como pertenecientes en primera instancia al grupo químico del ácido fosfónico y, en segunda instancia, como organofosforados (4143). Debido a que los herbicidas organofosforados son estructuralmente diferentes a los insecticidas de este mismo grupo químico y su poder inhibitorio de la acetilcolinesterasa es muy débil, no son relevantes desde el punto de vista toxicológico (24). Por estas razones, en el presente estudio se clasifican en un grupo químico diferente al de los organofosforados (cuadro 3).

El control de la exposición a plaguicidas en nuestro medio es difícil, considerando que la mayoría de trabajadores son de tipo informal y no cuentan con afiliación a una aseguradora de riesgos profesionales, ni con un plan de atención básico que evalúe periódicamente su estado de salud y sus niveles de actividad de la acetilcolinesterasa. Ante esta situación, son importantes los programas de capacitación y las campañas de sensibilización sobre los efectos de los plaguicidas en la salud, las medidas de higiene y seguridad en el trabajo, y el uso correcto de los elementos de protección personal. Los trabajadores expuestos a plaguicidas usualmente requieren de un equipo de protección personal que incluya respirador, chaqueta $y$ pantalón impermeables, guantes y botas (7).

Sólo $16,6 \%$ de los trabajadores que presentaron niveles anormales de acetilcolinesterasa, refirieron haber recibido capacitación sobre el uso y manejo de plaguicidas, resultados que sugieren deficiencia en este tipo de programas y en las campañas de sensibilización que sobre estos temas realizan las empresas. Esto puede indicar que muchos participantes no son conscientes del riesgo al que están expuestos, lo cual se aprecia en la deficiencia de medidas de higiene y seguridad, y el poco uso del equipo de protección personal, lo que puede facilitar la exposición a plaguicidas, tanto en el lugar de trabajo como en sus hogares.

Ningún participante reportó el uso del equipo de protección personal completo; sólo utilizaban algunos de sus elementos y, si se considera que la inhalación es la principal vía de absorción de estas sustancias, fueron pocos los que refirieron el uso de respiradores (5,3\%). Hay que tener en cuenta que esta forma de utilizar los elementos 
de protección personal puede generar hábitos de falsa protección frente al riesgo. Por otra parte, aunque los equipo de protección personal y las medidas de higiene y seguridad en el trabajo son necesarias para la prevención de los accidentes de trabajo y de las intoxicaciones agudas, también se debe tener presente que no son suficientes para proteger a los trabajadores de los riesgos que generan las exposiciones crónicas (44).

Como se había previsto en una publicación anterior del Programa VEO (22), el seguimiento de las determinaciones de acetilcolinesterasa quedó reducido a los controles de los participantes con valores anormales de actividad de la enzima, debido a la crisis económica de las entidades territoriales de salud, al cambio, despido y disminución del personal con experiencia en el Programa VEO, y a los problemas de orden público.

Por otra parte, con respecto al manejo de prevención y control, es importante señalar que, aunque la afiliación al Sistema General de Seguridad Social ha aumentado significativamente en el reporte del VEO, pasando de 31,7\% en el período de 1993-1995 a $76,2 \%$ en el presente estudio, la prevalencia de anormalidades de actividad de la acetilcolinesterasa se ha ido incrementando y, según lo reportado por los participantes con valores anormales, en muchos casos las medidas correctivas no se aplican o son insuficientes. Con el propósito de detectar los niveles de recuperación de actividad de la enzima, los controles deben realizarse repitiendo la prueba de acetilcolinesterasa quince días después de haber sido retirado el trabajador de la exposición; sin embargo, una gran proporción de participantes manifiesta que no se han cumplido estas condiciones, lo cual sugiere que hay casos no detectados, diagnósticos tardíos y deficiencias en el manejo del paciente.

El incremento en la prevalencia de valores anormales de acetilcolinesterasa en el Programa VEO es una manifestación de que no se están dando condiciones que favorezcan la seguridad ocupacional niambiental en el uso de los plaguicidas, en gran parte de las regiones estudiadas. Para que los trabajadores como los incluidos en el presente estudio obtengan seguridad laboral, se necesitan cambios en: las condiciones generales de trabajo, como dotación completa de equipos de protección personal y rotación a puestos de trabajo con menor riesgo de exposición; la atención médica, como afiliaciónaadministradorasderiesgosprofesionales, controles médicos periódicos y seguimiento de los niveles de actividad de la acetlcolinesterasa; los métodos de control de plagas, que prioricen formas de menor riesgo para la salud de la población, como sistemas naturales de control y disminución en el uso y comercialización de plaguicidas de alto riesgo; además, efectivas campañas sanitarias y de capacitación que orienten a los trabajadores en el uso y manejo de los plaguicidas, y en las medidas de higiene y seguridad en el trabajo.

Una de las limitaciones en el análisis de la presente información ha sido el reporte de datos en formatos de encuesta modificados por algunas entidades territoriales de salud según sus consideraciones particulares. Debido a que estas modificaciones dificultan y entorpecen el análisis, estamos solicitando la colaboración de las secretarías de salud para que no alteren los formatos suministrados por el Instituto Nacional de Salud, de manera que se pueda aprovechar al máximo y con mayor eficiencia la información recolectada y el trabajo realizado por los funcionarios que colaboran con el Programa VEO.

Igualmente, aprovechamos la presente publicación para extender una invitación a las secretarías de salud que no están participando o que han participado en forma interrumpida en el Programa VEO, para que se inscriban y así se logre valorar en el país la problemática de las intoxicaciones por plaguicidas en sus verdaderas dimensiones.

\section{Agradecimientos}

De manera especial, los autores agradecen a los trabajadores y participantes, a los gerentes de empresas y propietarios de fincas, a los coordinadores del Programa VEO en cada una de las entidades territoriales de salud participantes, así como a los supervisores y técnicos de saneamiento; de igual manera, a las directivas de hospitales y secretarías de salud por el apoyo brindado en la realización del programa.

\section{Conflicto de intereses}

Los autores declaramos que durante la realización del trabajo no existió conflicto de intereses de ningún tipo.

\section{Financiación}

Este trabajo fue financiado por el Plan de Atención Básico de cada una de las Entidades Territoriales de Salud participantes en el Programa VEO durante el período de 2002 a 2005 y por el Grupo de Salud Ambiental de la Subdirección Red Nacional de Laboratorios, del Instituto Nacional de Salud. 


\section{Referencias}

1. Anwar WA. Biomarkers of human exposure to pesticides. Environ Health Perspect. 1997;105(Suppl.4):801-6.

2. Rodríguez CA. Jornadas Internacionales Multidisciplinarias y Tripartitas. Agro: Trabajo y Salud. Resistencia, Chaco, Argentina. 14, 15 y 16 de agosto de 1999. Fecha de consulta: 10 de enero de 2008. Disponible en: http://www. cepis.org.pe/eswww/ocupfile/plaguicidas.doc.

3. Petrelli G, Siepi G, Miligi L, Vineis P. Solvents in pesticides. Scand J Work Environ Health. 1993;19:63-54.

4. Henao S, Corey G. Plaguicidas inhibidores de las colinesterasas. Serie de Vigilancia 11. Metepec, México: Asistencia Editorial de Clemente Aguilar; 1991. p. 17-169.

5. Schosinsky K, Quintana E. Detección de plaguicidas en vegetales de Costa Rica mediante la inhibición de colinesterasas humanas. Arch Latinoam Nutr. 2004;54:1-10.

6. Nivia E. Los plaguicidas en Colombia. Revista Semillas. 2004;21. Fecha de consulta: noviembre de 2007. Disponible en: http://www.semillas.org.co/sitio.shtml?apc=e1b-30353$30353 \& x=20154645$.

7. Jaga K, Dharmani C. Sources of exposure and public health implications of organophosphate pesticides. Pan Am J Public Health. 2003;14:171-85.

8. Rendón von Osten J, Epomex C, Tinoco-Ojanguren R, Soares AM, Guilhermino L. Effect of pesticide exposure on acetylcholinesterase activity in subsistence farmers from Campeche, Mexico. Arch Environ Health. 2004;59:418-25.

9. Ministerio de Agricultura y Desarrollo Rural, Ministerio de Ambiente, Vivienda y Desarrollo Territorial, Ministerio de Educación Nacional, Ministerio de Comercio, Industria, y Turismo, Ministerio de Minas y Energía, Ministerio de la Protección Social, et al. Lineamientos para la formulación de la política integral de salud ambiental con énfasis en los componentes de calidad de aire, calidad de agua y seguridad química. Documento Conpes 3550. Bogotá D.C.: Departamento Nacional de Planeación; 2008. p. 2-47.

10. CEPIS-OPS-OMS. Toxicología/Cursos de autoinstrucción, diagnóstico, tratamiento y prevención de intoxicaciones agudas por plaguicidas. Fecha de consulta: noviembre 2007. Disponible en: http://www.cepis.ops-oms.org/ tutorial2/e/bienvenida.html

11. García JE. Intoxicaciones agudas con plaguicidas: costos humanos y económicos. Rev Panam Salud Pública. 1998;4:383-7.

12. Palacios ME, Paz P, Hernández S, Mendoza L. Sintomatología persistente en trabajadores industrialmente expuestos a plaguicidas organofosforados. Salud Pública Mex. 1999;41:55-61.

13. Palacios ME. Evaluación de organofosforados. Salud Pública Mex. 1999;41:55-61.

14. DANE-SISAC. Síntesis Encuesta Nacional Agropecuaria, ENA 2004. Fecha de consulta: noviembre de 2007. Disponible en: http://amedea.eu/files/ena_2004.pdf.

15. Ministerio de Agricultura y Desarrollo Rural, Instituto Colombiano Agropecuario. Comercialización de plaguicidas 2002. Producción-ventas, importación-exportación. Bogotá: Editorial Produmedios; 2005. p. 8, 18-21.
16. Ministerio de Agricultura y Desarrollo Rural, Instituto Colombiano Agropecuario. Estadísticas de comercialización de plaguicidas 2005. Bogotá, D.C.: Instituto Colombiano Agropecuario; 2005. p. 6, 32-4.

17. Morgan DP. Diagnóstico y tratamiento de los envenenamientos con plaguicidas. lowa Pesticide Hazard Assessment Project. Tercera edición. lowa City, lowa: University of lowa Medical School; 1992.

18. Ministerio de Salud. Uso y manejo de plaguicidas. Decreto número 1843 de 1991; Bogotá: Ministerio de Salud; 1991. p. 5-47.

19. Instituto Nacional de Salud. Estadísticas de la Vigilancia. Tablas de Notificación. Sistema de Vigilancia en Salud Pública-Sivigila. Fecha de consulta: noviembre de 2007. Disponible en: http://www.ins.gov.co/?idcategoria=1729.

20. Ministerio de Salud. Programa de vigilancia epidemiológica. Resolución Número 4547 de 1998. Bogotá, D.C.: Ministerio de Salud; 1998.

21. Varona M, Morales L, Ortiz J, Sánchez JF, Cárdenas O, De la Hoz F. Panorama epidemiológico de exposición a plaguicidas inhibidores de colinesterasa en 17 departamentos del país. Biomédica. 1998;18:22-9.

22. Silva E, Morales L, Ortiz J. Evaluación epidemiológica de plaguicidas inhibidores de acetilcolinesterasa en Colombia, 1996-1997. Biomédica. 2000;20:200-9.

23. Cárdenas O, Silva E, Morales L, Ortiz JE. Estudio epidemiológicodeexposiciónaplaguicidasorganofosforados y carbamatos en siete departamentos colombianos, 19982001. Biomédica. 2005;25:170-80

24. Edson EF, Fenwick ML. Measurement of cholinesterase activity of whole blood. Brit Med J. 1955;1:1218.

25. Wilson BW, Sanborn JR, O'Malley MO, Henderson JD, Billitti JR. Monitoring the pesticide-exposed worker. Occupational Medicine. Seattle, Washington: Editorial Harborview Medical Center; 1998. p. 47-363.

26. Fillmore CM, Lessenger JE. A cholinesterase testing program for pesticide applicators. J Occup Med. 1993;35: 61-70.

27. Limperos G, Ranta KE. A rapid screening test for the determination of the approximate cholinesterase activity of human blood. Science. 1953;117:453-5.

28. Ministerio de Salud Costa Rica, Seguro Social de Costa Rica, INCIENSA. Sistema Nacional de Vigilancia de la Salud de Costa Rica. Boletín Epidemiológico. 2003;3:1-2.

29. Red de Acción en Plaguicidas de América Latina (RAPAL). Los niños y los plaguicidas. Quito: Enlace, Editorial Fundación Natura; 1988. p. 8.

30. Marín J, Toledo R, Corriols M. Análisis de la situación de salud de Nicaragua. Efectos adversos a la salud causados por la exposición a plaguicidas 1990-1997. Nicaragua: OPS; 2007. p. 1-8.

31. Vindas R, Ortiz F, Ramírez V, Cuenca P. Genotoxicidad de tres plaguicidas utilizados en la actividad bananera de Costa Rica. Rev Biol Trop. 2004;52:1-14.

32. Silva E, Morales L, Ortiz JE. Evaluación epidemiológica de plaguicidas en el departamento del Meta, Programa VEO, 1996 a 1997. Inf Quin Epidemiol Nac. 2002;7:389-401. 
33. Morales L, Silva E, Ramírez P, García A, Ortíz J. Intoxicación por plaguicidas en el departamento del Valle del Cauca. Inf Quin Epidemiol Nac. 1998;3:222-4.

34. Idrovo AJ. Intoxicaciones masivas con plaguicidas en Colombia. Biomédica. 1999;19:67-76.

35. Palacios ME. Aplicación de un instrumento para evaluar exposición a plaguicidas organofosforados, efectos agudos y subagudos en la salud de trabajadores agrícolas. Rev Fac Med UNAM. 2003;46:22-7.

36. Instituto Nacional de Salud. Sistema de Vigilancia en Salud Pública-SIVIGILA. Proyecto intercambio de experiencias en el control de riesgos ocasionados por plaguicidas El Salvador-Colombia, octubre 2002-noviembre 2003 - I parte. Boletín Epidemiológico Semanal. Bogotá, D.C.: INS; 2003. p. 1-9.

37. Organización Panamericana de la Salud, Organización Mundial de la Salud, Area de Desarrollo Sostenible y Salud Ambiental, Agencia Danesa para el Desarrollo Internacional. Proyecto Aspectos Ocupacionales y Ambientales de la Exposición a Plaguicidas en el Istmo Centroamericano (PLAGSALUD). Fecha de consulta: enero de 2008. Disponible en: http://www2.ops.org/sv/plagsalud/ index.htm

38. Consejo de Estado. Endosulfán sí es peligroso y está prohibido en Colombia. Comunicado para publicación abril 2 de 2001. Bogotá, D.C.: Consejo de Estado; 2001.
39. RAP-AL. Un muerto y 154 personas intoxicadas con endosulfán, cuyo uso está prohibido. Agrotóxicos. Colombia 24 de agosto de 2007. Fecha de consulta: julio de 2009. Disponible en: http://www.rel-uita.org/agricultura/ agrotoxicos/colombia_muerto_endosulfan.htm.

40. Terratoxnews Latinoamérica. Colombia: mortífero uso de plaguicida prohibido "endosulfán". El Diario de Otún. 03 agosto de 2007. Fecha de consulta: julio de 2009. Disponible en: http://www.eldiario.com.co/bodyindex.php? anteriores $=2007-08-04 \&$ id noticia $=91864 \&$ nombre $=$ noticia s\&padre $=97$

41. Jeyaratnam J, Maroni M. Organophosphorous compounds. Toxicology. 1994;91:15-27.

42. Worthing CR, Hance RJ. Glyphosate. The Pesticide Manual. A World Compendium. 9th edition. Surrey, U.K: The British Crop Protection Council; 1991. p. 459-60.

43. SATA. Guía para la Protección y Nutrición Vegetal. Glifosato - glyphosate. Fecha de consulta: septiembre de 2009. Disponible en http://www.laguiasata.com/Glifosato.html.

44. Schosinsky K, Quintana E. Detección de plaguicidas en vegetales de Costa Rica mediante la inhibición de colinesterasas humanas. Arch Latinoam Nutr. 2004;54:444-8. 